\title{
On $k$-Fibonacci balancing and $k$-Fibonacci Lucas-balancing numbers
}

\section{Rihane S.E.}

The balancing number $n$ and the balancer $r$ are solution of the Diophantine equation

$$
1+2+\cdots+(n-1)=(n+1)+(n+2)+\cdots+(n+r) .
$$

It is well known that if $n$ is balancing number, then $8 n^{2}+1$ is a perfect square and its positive square root is called a Lucas-balancing number. For an integer $k \geq 2$, let $\left(F_{n}^{(k)}\right)_{n}$ be the $k$-generalized Fibonacci sequence, which starts with $0, \ldots, 0,1,1$ ( $k$ terms) and each term afterwards is the sum of the $k$ preceding terms. The purpose of this paper is to show that 1,6930 are the only balancing numbers and 1, 3 are the only Lucas-balancing numbers, which are a term of $k$-generalized Fibonacci sequence. This generalizes the result from [Fibonacci Quart. 2004, 42 (4), 330-340].

Key words and phrases: $k$-generalized Fibonacci numbers, balancing numbers, Lucas-balancing numbers, linear form in logarithms, reduction method.

Department of Mathematics, Institute of Science and Technology, University Center of Mila, Mila, Algeria

E-mail: salahrihane@hotmail.fr

\section{Introduction}

The first definition of balancing numbers is essentially due to R.P. Finkelstein [8], although he called them numerical centers. A positive integer $n$ is called balancing number if

$$
1+2+\cdots+(n-1)=(n+1)+(n+2)+\cdots+(n+r)
$$

holds for some positive integer $r$. Then $r$ is called balancer corresponding to the balancing number $n$. The $n$-th term of the sequence of balancing numbers is denoted by $B_{n}$. A. Behera and G.K. Panda [2] proved that the balancing numbers fulfill the recurrence relation

$$
B_{0}=1, \quad B_{1}=6, \quad B_{n}=6 B_{n-1}-B_{n-2} \quad \text { for all } n \geq 2 .
$$

It is well known that if $n$ is a balancing number, then $8 n^{2}+1$ is a perfect square, and the positive square root of $8 n^{2}+1$ is called a Lucas-balancing number which is denoted by $C_{n}$ (see [13]). The Lucas-balancing numbers $C_{n}$ satisfy the recurrence relation

$$
C_{0}=1, \quad C_{1}=3, \quad C_{n}=6 C_{n-1}-C_{n-2} \text { for all } n \geq 2 .
$$

The balancing and Lucas-balancing numbers are indexed in The On-Line Encyclopedia of Integer Sequences (OEIS) as A001109 and A001541, respectively. 
The Fibonacci sequence $\left(F_{n}\right)_{n \geq 0}$ is given by

$$
F_{0}=0, \quad F_{1}=1, \quad F_{n}=F_{n-1}+F_{n-2} \quad \text { for all } n \geq 2 .
$$

It is the sequence A000045 in OEIS.

A balancing number is called Fibonacci balancing number if it is a Fibonacci number (see [9]). In [9], K. Liptai has shown that 1 is the only Fibonacci balancing number.

Let $k \geq 2$ be an integer. We consider a generalization of Fibonacci sequence called the $k$-generalized Fibonacci sequence $F_{n}^{(k)}$ defined as

$$
F_{n}^{(k)}=F_{n-1}^{(k)}+F_{n-2}^{(k)}+\cdots+F_{n-k}^{(k)} \text { for all } n \geq 2,
$$

with the initial conditions $F_{-(k-2)}^{(k)}=F_{-(k-3)}^{(k)}=\cdots=F_{0}^{(k)}=0$ and $F_{1}^{(k)}=1$. If $k=2$, we obtain the classical Fibonacci sequence. Below we present the values of these numbers for the first few values of $k$ and $n \geq 1$. Note that the underlying terms are balancing or Lucas-balancing numbers.

\begin{tabular}{cll}
\hline$k$ & Name & First non-zero terms \\
\hline 2 & Fibonacci & $\underline{1}, \underline{1}, 2, \underline{3}, 5,8,13,21,34,55,89,144,233,377,610, \ldots$ \\
3 & Tribonacci & $\underline{1}, \underline{1}, 2,4,7,13,24,44,81,149,274,504,927,1705,3136, \ldots$ \\
4 & Tetranacci & $\underline{1}, \underline{1}, 2,4,8,15,29,56,108,208,401,773,1490,2872,5536, \ldots$ \\
5 & Pentanacci & $\underline{1}, \underline{1}, 2,4,8,16,31,61,120,236,464,912,1793,3525, \underline{6930} \ldots$ \\
6 & Hexanacci & $\underline{1}, \underline{1}, 2,4,8,16,32,63,125,248,492,976,1936,3840,7617, \ldots$ \\
7 & Heptanacci & $\underline{1}, \underline{1}, 2,4,8,16,32,64,127,253,504,1004,2000,3984,7936, \ldots$ \\
8 & Octanacci & $\underline{1}, \underline{1}, 2,4,8,16,32,64,128,255,509,1016,2028,4048,8080, \ldots$ \\
9 & Nonanacci & $\underline{1}, \underline{1}, 2,4,8,16,32,64,128,256,511,1021,2040,4076,8144, \ldots$ \\
10 & Decanacci & $\underline{1}, \underline{1}, 2,4,8,16,32,64,128,256,512,1023,2045,4088,8172, \ldots$ \\
\hline
\end{tabular}

We say that a balancing number (Lucas-balancing number) is $k$-Fibonacci balancing number ( $k$-Fibonacci Lucas-balancing number) if it is $k$-Fibonacci number too. The aim of the present work is to determine all the $k$-Fibonacci balancing and $k$-Fibonacci Lucas-balancing numbers. We prove the following results.

Theorem 1. 1 and 6930 are the only k-Fibonacci balancing number. Moreover, all the solutions of the Diophantine equation

$$
F_{n}^{(k)}=B_{m}
$$

are given by $(n, k, m)=(1, k, 0),(2, k, 0),(15,5,6)$.

Theorem 2. 1 and 3 are the only k-Fibonacci Lucas-balancing number. Moreover, all the solutions of the Diophantine equation

$$
F_{n}^{(k)}=C_{m}
$$

are given by $(n, k, m)=(1, k, 0),(2, k, 0),(4,2,1)$.

Our proofs of Theorems 1 and 2 are mainly based on linear forms in logarithms of algebraic numbers and a reduction algorithm originaly introduced by A. Baker and H. Davenport in [1]. Here, we use a version due to A. Dujella and A. Pethó in [7, Lemma 5 (a)]. 


\section{Premilmeries and known results}

This section is devoted to collect a few definitions, notations and theorems, which will be used in the rest of this work.

\subsection{Linear forms in logarithms}

For any non-zero algebraic number $\eta$ of degree $d$ over $\mathbb{Q}$, whose minimal polynomial over $\mathbb{Z}$ is $a \prod_{j=1}^{d}\left(X-\eta^{(j)}\right)$, we denote by

$$
h(\eta)=\frac{1}{d}\left(\log |a|+\sum_{j=1}^{d} \log \max \left\{1,\left|\eta^{(j)}\right|\right\}\right)
$$

the usual absolute logarithmic height of $\eta$. In particular, if $\eta=p / q$ is a rational number with $\operatorname{gcd}(p, q)=1$ and $q>0$, then $h(\eta)=\log \max \{|p|, q\}$. The following properties of the logarithmic height function $h(\cdot)$, which will be used in the next sections without special reference, are also known:

$$
\begin{aligned}
h(\eta \pm \gamma) & \leq h(\eta)+h(\gamma)+\log 2 \\
h\left(\eta \gamma^{ \pm 1}\right) & \leq h(\eta)+h(\gamma) \\
h\left(\eta^{s}\right) & =|s| h(\eta), \quad s \in \mathbb{Z} .
\end{aligned}
$$

The main approach to show Theorems 1 and 2 is the Baker's theory about lower bounds for linear forms in logarithms. In [10], E.M. Matveev proved the following theorem.

Theorem 3 ([10]). Let $\eta_{1}, \ldots, \eta_{s}$ be a real algebraic numbers and let $b_{1}, \ldots, b_{s}$ be nonzero rational integer numbers. Let $d_{\mathbb{K}}$ be the degree of the number field $\mathbb{Q}\left(\eta_{1}, \ldots, \eta_{s}\right)$ over $\mathbb{Q}$. Define

$$
\Gamma:=\eta_{1}^{b_{1}} \cdots \eta_{s}^{b_{s}}-1
$$

If $\Gamma \neq 0$, then

$$
|\Gamma| \geq \exp \left(-1.4 \cdot 30^{s+3} s^{4.5} d_{\mathbb{K}}^{2}\left(1+\log d_{\mathbb{K}}\right)(1+\log B) A_{1} \cdots A_{s}\right),
$$

where $A_{j}=\max \left\{d_{\mathbb{K}} h(\eta),|\log \eta|, 0.16\right\}$ for $j=1, \ldots, s$, and $B \geq \max \left\{\left|b_{1}\right|, \ldots,\left|b_{s}\right|\right\}$.

\subsection{The de Weger reduction algorithm}

Here, we present a variant of the reduction method of Baker and Davenport due to de Weger [14].

Let $\vartheta_{1}, \vartheta_{2}, \beta \in \mathbb{R}$ be given and let $x_{1}, x_{2} \in \mathbb{Z}$ be unknowns. Let

$$
\Lambda=\beta+x_{1} \vartheta_{1}+x_{2} \vartheta_{2} \text {. }
$$

Set $X=\max \left\{\left|x_{1}\right|,\left|x_{2}\right|\right\}$. Let $X_{0}, Y$ be positive. Assume that

$$
|\Lambda|<c \exp (-\rho Y)
$$

and

$$
Y \leq X \leq X_{0}
$$

where $c, \rho$ be positive constants.

When $\beta=0$ in (5), we get $\Lambda=x_{1} \vartheta_{1}+x_{2} \vartheta_{2}$. Put $\vartheta=-\vartheta_{1} / \vartheta_{2}$. We assume that $x_{1}$ and $x_{2}$ are coprime. Let the continued fraction expansion of $\vartheta$ be given by $\left[a_{0}, a_{1}, a_{2}, \ldots\right]$, and let the $k$ th convergent of $\vartheta$ be $p_{k} / q_{k}$ for $k=0,1,2, \ldots$. We may assume without loss of generality that $\left|\vartheta_{1}\right|<\left|\vartheta_{2}\right|$ and that $x_{1}>0$. We have the following results. 
Lemma 1 ([14, Lemma 3.1]). If (6) and (7) hold for $x_{1}, x_{2}$ with $X \geq 1$ and $\beta=0$, then $\left(-x_{2}, x_{1}\right)=\left(p_{k}, q_{k}\right)$ for an index $k$ that satisfies

$$
k \leq-1+\frac{\log \left(1+X_{0} \sqrt{5}\right)}{\log \left(\frac{1+\sqrt{5}}{2}\right)}:=Y_{0} .
$$

Lemma 2 ([14, Lemma 3.2]). Let $A=\max _{0 \leq k \leq \gamma_{0}} a_{k+1}$. If (6) and (7) hold for $x_{1}, x_{2}$ with $X \geq 1$ and $\beta=0$, then

$$
Y<\frac{1}{\rho} \log \left(\frac{c(A+2)}{\left|\vartheta_{2}\right|}\right)+\frac{1}{\rho} \log X<\frac{1}{\rho} \log \left(\frac{c(A+2) X_{0}}{\left|\vartheta_{2}\right|}\right) .
$$

When $\beta \neq 0$ in (5), put $\vartheta=-\vartheta_{1} / \vartheta_{2}$ and $\psi=\beta / \vartheta_{2}$. Then we have $\frac{\Lambda}{\vartheta_{2}}=\psi-x_{1} \vartheta+x_{2}$. Let $p / q$ be a convergent of $\vartheta$ with $q>X_{0}$. For a real number $x$ we let

$$
\|x\|=\min \{|x-n|: n \in \mathbb{Z}\}
$$

be the distance from $x$ to the nearest integer. We have the following result.

Lemma 3 ([14, Lemma 3.3]). Suppose that $\|q \psi\|>\frac{2 X_{0}}{q}$. Then, the solutions of (6) and (7) satisfy

$$
Y<\frac{1}{\rho} \log \left(\frac{q^{2} c}{\left|\vartheta_{2}\right| X_{0}}\right) .
$$

\subsection{The balancing and Lucas-balancing sequence}

Let $\delta:=(3+2 \sqrt{2})$ and $\bar{\delta}:=(3-2 \sqrt{2})$ be the roots of the characteristic equation $x^{2}-6 x+1$ of both the balancing and Lucas-balancing sequences, the Binet formulas

$$
B_{n}=\frac{\delta^{n}-\bar{\delta}^{n}}{4 \sqrt{2}}
$$

and

$$
C_{n}=\frac{\delta^{n}+\bar{\delta}^{n}}{2}
$$

hold for all nonnegative integer $n$ 's. Furthermore, the inequalities

$$
\delta^{n-1}<B_{n}<\delta^{n}
$$

and

$$
\delta^{n-1}<C_{n}<\delta^{n}
$$

hold for all $n \geq 1$.

\subsection{Properties of $k$-generalized Fibonacci sequence}

In this subsection, we recall some facts and properties of the $k$-generalized Fibonacci sequence which will be used later. The characteristic polynomial of the $k$-generalized Fibonacci numbers $\left(F_{n}^{(k)}\right)_{n}$ is

$$
\Psi_{k}(x)=x^{k}-x^{k-1}-\cdots-x-1 .
$$


$\Psi_{k}(x)$ is irreducible over $\mathrm{Q}[x]$ and has just one root $\alpha(k)$ outside the unit circle (see, for example, $[11,12,15])$. It is real and positive, so it satisfies $\alpha(k)>1$. The other root are strictly inside the unit circle. Furthermore, in [15] D.A. Wolfram showed that

$$
2\left(1-2^{-k}\right)<\alpha(k)<2 \text { for all } k \geq 2 .
$$

To simplify the notation, in general, we omit the dependence on $k$ of $\phi$. For $s \geq 2$, let

$$
f_{s}(x):=\frac{x-1}{2+(s+1)(x-2)} .
$$

In [6], G.P.B. Dresden, Z. Du gave the Binet-type formula

$$
F_{n}^{(k)}=\sum_{i=1}^{k} f_{k}\left(\alpha_{i}\right) \alpha_{i}^{n-1},
$$

where $\alpha_{i}$ are the zeros of $\Psi_{k}(x)$, and proved that

$$
\left|F_{n}^{(k)}-f_{k}(\alpha) \alpha^{n-1}\right|<\frac{1}{2}
$$

hold for all $n \geq k-2$. Furthermore, it was showed in [3] that

$$
\alpha^{n-2} \leq F_{n}^{(k)} \leq \alpha^{n-1}
$$

hold for all $n \geq 1$.

In [4], J.J. Bravo, C.A. Gómez and F. Luca proved that $1 / 2<f_{k}(\alpha)<3 / 4$ and $\left|f_{k}\left(\alpha_{i}\right)\right|<1$, $2 \leq i \leq k$, hold. So, the number $f_{k}(\alpha)$ is not an algebraic integer. In addition, they proved that the logarithmic height of $f$ satisfies

$$
h\left(f_{k}(\alpha)\right)<\log (k+1)+\log 4 \text { for all } k \geq 2 .
$$

Finally, in [5, pp. 542,543] the authors proved that for all $n \geq k+2$ we have

$$
F_{n}^{(k)}=2^{n-2}(1+\zeta), \quad \text { where }|\zeta|<\frac{1}{2^{k / 2}} .
$$

\section{$3 \quad k$-Fibonacci balancing numbers}

This section is devoted to show Theorem 1.

\subsection{An inequality for $n$ and $m$ versus $k$}

If $2 \leq n \leq k+1$, we have $F_{n}^{(k)}=2^{n-2}$ and since 1 is the only perfect power in the balancing sequence, we deduce that equation (1) has only the solution $(n, k, m)=(2, k, 0)$ in this range. The fact that $F_{1}^{(k)}=F_{2}^{(k)}$ imply that $(1, k, 0)$ is also a solution of the Diophantine equation (1). From now, we assume that $n \geq k+2$. Further we may suppose that $k \geq 3$ because that case $k=2$ is already studied.

Using inequalities (14) and (10), we get from equation (1) that

$$
\alpha^{n-2} \leq \delta^{m-1} \quad \text { and } \quad \delta^{m-2} \leq \alpha^{n-1} .
$$

The above inequalities give

$$
(n-2)\left(\frac{\log \alpha}{\log \delta}\right)+1 \leq m \leq(n-1)\left(\frac{\log \alpha}{\log \delta}\right)+2 .
$$

Using the fact that $7 / 4<\alpha<2$ for all $k \geq 3$ (see (12)), we deduce that

$$
0.3 n-0.6<m<0.4 n+1.7 \text {. }
$$


Lemma 4. If $(n, k, m)$ is a solution in integers of equation (1) with $k \geq 3$ and $n \geq k+2$, then the inequalities $2.4 m<n<6.8 \cdot 10^{15} k^{4} \log ^{3} k$ hold.

Proof. From equation (1), estimate (13) and identity (8), we have

$$
\left|f_{k}(\alpha) \alpha^{n-1}-\frac{\delta^{m}}{4 \sqrt{2}}\right|<\frac{1}{2}+\frac{1}{4 \sqrt{2}} .
$$

If we multiply through by $4 \sqrt{2} \delta^{-m}$ we arrive at

$$
\left|\Gamma_{1}\right|<3.9 \delta^{-m}
$$

where $\Gamma_{1}=\left(4 \sqrt{2} f_{k}(\alpha)\right) \alpha^{n-1} \delta^{-m}-1$.

With the aim of applying Theorem 3 we choose

$$
\left(\eta_{1}, b_{1}\right):=\left(4 \sqrt{2} f_{k}(\alpha), 1\right), \quad\left(\eta_{2}, b_{2}\right):=(\alpha, n-1), \quad\left(\eta_{3}, b_{3}\right):=(\delta,-m) .
$$

For this choice, the field $\mathbb{K}:=\mathbb{Q}(\alpha, \sqrt{2})$ contains $\eta_{1}, \eta_{2}, \eta_{3}$ and has $d_{\mathbb{K}} \leq 2 k$. Since $h\left(\eta_{2}\right)=(\log \alpha) / k<(\log 2) / k$ and $h\left(\eta_{3}\right)=(\log \delta) / 2$, we deduce that

$$
\max \left\{2 k h\left(\eta_{2}\right),\left|\log \eta_{2}\right|, 0.16\right\}=2 \log 2:=A_{2}
$$

and

$$
\max \left\{2 k h\left(\eta_{3}\right),\left|\log \eta_{3}\right|, 0.16\right\}=k \log \delta:=A_{3} .
$$

On the other hand, by using the estimate (15) and the proprieties (3) together with (4), it follows that for all $k \geq 3$

$$
h\left(\eta_{1}\right) \leq h\left(f_{k}(\alpha)\right)+h(4 \sqrt{2})<\log (k+1)+\log 4+\log (4 \sqrt{2})<4.2 \log k .
$$

Thus, we obtain

$$
\max \left\{2 k h\left(\eta_{1}\right),\left|\log \eta_{1}\right|, 0.16\right\}<8.4 k \log k:=A_{1} .
$$

The fact that $0.4 n+1.7<n$ hold for all $n \geq 5$ and the inequality (17), imply that we can take $B:=n$.

Before applying Theorem 3 , we need to check that $\Gamma_{1} \neq 0$. Indeed, if we assume that $\Gamma_{1}=0$, we get that

$$
f_{k}(\alpha)=\frac{\delta^{m}}{4 \sqrt{2}} \alpha^{-n+1},
$$

and so $f_{k}(\alpha)$ would be an algebraic integer, contradicting some thing previously mentioned. Thus, $\Gamma_{1} \neq 0$. Therefore, by Theorem 3 , it result

$$
\left|\Gamma_{1}\right|>\exp \left(-1.432 \cdot 10^{11}(2 k)^{2}(1+\log (2 k))(1+\log n)(8.4 k \log k)(2 \log 2)(k \log \delta)\right) .
$$

When we compare the lower bound (19) and the upper bound (18) of $\left|\Gamma_{1}\right|$ we obtain

$$
m \log \delta-\log 3.9<1.18 \cdot 10^{13} k^{4} \log k(1+\log 2 k)(1+\log n),
$$

taking into account the facts $1+\log 2 k<2.6 \log k$ and $1+\log n<1.7 \log n$ which hold for $k \geq 3$ and $n \geq 5$, we conclude that $m<3 \cdot 10^{13} k^{4} \log ^{2} k \log n$. By the inequality (17), the last inequality becomes

$$
\frac{n}{\log n}<10^{14} k^{4} \log ^{2} k
$$


Since the function $x \mapsto x / \log x$ is increasing for all $x>e$, it is easy to check that

$$
\frac{x}{\log x}<T \quad \Longrightarrow \quad x<2 T \log T \quad \text { whenever } T \geq 3 \text {. }
$$

Thus, fixing $T:=10^{14} k^{4} \log ^{2} k$, inequality (20) together with $32.3+4 \log k+2 \log \log k<$ $34 \log k$, which holds for all $k \geq 2$, gives

$$
\begin{aligned}
n & <\left(2 \cdot 10^{14} k^{4} \log ^{2} k\right) \log \left(10^{14} k^{4} \log ^{2} k\right) \\
& <\left(2 \cdot 10^{14} k^{4} \log ^{2} k\right)(32.3+4 \log k+2 \log \log k)<6.8 \cdot 10^{15} k^{4} \log ^{3} k .
\end{aligned}
$$

Whence the result.

\subsection{The case $3 \leq k \leq 220$}

In this subsection, we treat the case $k \in[3,220]$. We show the following result.

Lemma 5. The Diophantine equation (1) has no solution, when $k \in[3,220]$ and $n \geq k+2$.

Proof. Let us set

$$
\Lambda_{1}=\log \left(\Gamma_{1}+1\right)=(n-1) \log \alpha-m \log \delta+\log \left(4 \sqrt{2} f_{k}(\alpha)\right) .
$$

Then, (18) can be rewritten as

$$
\left|e^{\Lambda_{1}}-1\right|<3.9 \delta^{-m}
$$

Note that $\Lambda_{1} \neq 0$, since $\Gamma_{1} \neq 0$, so we distinguish the following cases. If $\Lambda_{1}>0$, then $e^{\Lambda_{1}}-1>0$. Using the fact that $x \leq e^{x}-1$ for all $x \in \mathbb{R}$, from (22) we obtain $0<\Lambda_{1}<3.9 \delta^{-m}$. Now, if $\Lambda_{1}<0$, it is easy to see that $3.9 \delta^{-m}<1 / 2$ holds for all $m \geq 4$. Thus, from (22) we have that $\left|e^{\Lambda_{1}}-1\right|<1 / 2$ and therefore $e^{\left|\Lambda_{1}\right|}<2$. Since $\Lambda_{1}<0$, we have

$$
0<\left|\Lambda_{1}\right| \leq e^{\left|\Lambda_{1}\right|}-1=e^{\left|\Lambda_{1}\right|}\left|e^{\Lambda_{1}}-1\right|<7.8 \delta^{-m}
$$

Hence, in both cases one has

$$
0<\left|\Lambda_{1}\right|<7.8 \delta^{-m}
$$

In order to apply Lemma 3, we fix

$$
\begin{gathered}
c:=7.8, \quad \rho:=1.76, \quad \psi:=\frac{\log \left(4 \sqrt{2} f_{k}(\alpha)\right)}{\log \delta}, \\
\vartheta:=\frac{\log \delta}{\log \alpha}, \quad \vartheta_{1}:=-\log \delta, \quad \vartheta_{2}:=\log \alpha, \quad \beta:=\log \left(4 \sqrt{2} f_{k}(\alpha)\right) .
\end{gathered}
$$

For each $k \in[3,220]$, we find a good approximation of $\alpha$ and a convergent $p_{\ell} / q_{\ell}$ of the continued fraction of $\vartheta$ such that $q_{\ell}>X_{0}$, where $X_{0}=\left\lfloor 6.8 \cdot 10^{15} k^{4} \log ^{3} k\right\rfloor$, which is an upper bound of $\max \{n-1, m\}$ from Lemma 4 . After doing this, we use Lemma 3 on inequality (23). A computer search with Mathematica revealed that the maximum value of $\left\lfloor\frac{1}{\delta} \log \left(q^{2} c /\left|\vartheta_{2}\right| X_{0}\right)\right\rfloor$ over all $k \in[3,220]$ is $45.6224 \ldots$, which according to Lemma 3 , is an upper bound on $m$. Hence, we deduce that the possible solutions $(m, n, k)$ of the equation (1) for which $k \in[3,220]$ have $m \leq 45$, therefore we use inequalities (17) to obtain $n \leq 151$.

Finally, we used Mathematica to compare $F_{n}^{(k)}$ and $B_{m}$ for the range $5 \leq n \leq 151$ and $2 \leq m \leq 45$, with $m<n / 2.4$ and checked that the only solution of the equation (1) is $6930=B_{6}=F_{15}^{(5)}$. 


\subsection{The case $k>220$}

In this subsection, we analyze the case $k>220$.

Lemma 6. The Diophantine equation (1) has no solution when $k>220$ and $n \geq k+2$.

Proof. For $k>220$ we have $2.4 m<n<6.8 \cdot 10^{15} k^{4} \log ^{3} k<2^{k / 2}$. Using (8) and (16), we express the equation (1) as

$$
2^{n-2}-\frac{\delta^{m}}{4 \sqrt{2}}=2^{n-2} \zeta-\frac{\bar{\delta}^{m}}{4 \sqrt{2}^{2}}
$$

by taking absolute value we obtain

$$
\left|2^{n-2}-\frac{\delta^{m}}{4 \sqrt{2}}\right|<\frac{2^{n-2}}{2^{k / 2}}+\frac{1}{4 \sqrt{2}}
$$

which gives

$$
\left|1-(\sqrt{2})^{-1} 2^{-n} \delta^{m}\right|<\frac{1.1}{2^{k / 2}}
$$

where we have used the fact $1 /\left(\sqrt{2} \cdot 2^{n}\right)<0.1 / 2^{k / 2}$, because $n \geq k+2$. We will apply Theorem 3 to obtain a lower bound to the left-hand side of inequality (24). Choose

$$
t:=3, \quad\left(\eta_{1}, b_{1}\right):=(\sqrt{2},-1), \quad\left(\eta_{2}, b_{2}\right):=(2,-n), \quad\left(\eta_{3}, b_{3}\right):=(\delta, m) .
$$

Since $\eta_{1}, \eta_{2}, \eta_{3} \in \mathbb{K}:=\mathbb{Q}(\sqrt{2})$, then $d_{\mathbb{K}}=2$. The left-hand side of (24) is not zero. Indeed, if this is zero, we would then get that $\delta^{2 m}$ is a rational numbers, which is impossible for all positive integers $m$.

We can choose $B:=n$, because $m \leq n$. On the other hand, since

$$
h\left(\eta_{1}\right)=\log (\sqrt{2}), \quad h\left(\eta_{2}\right)=\log 2, \quad h\left(\eta_{3}\right)=(\log \delta) / 2,
$$

we deduce that

$$
\max \left\{2 h\left(\eta_{1}\right),\left|\log \eta_{1}\right|, 0.16\right\}=\log 2:=A_{1}, \quad \max \left\{2 h\left(\eta_{2}\right),\left|\log \eta_{2}\right|, 0.16\right\}=2 \log 2:=A_{2}
$$

and

$$
\max \left\{2 h\left(\eta_{3}\right),\left|\log \eta_{3}\right|, 0.16\right\}=\log \delta:=A_{3}
$$

Therefore, according to Theorem 3 we have

$$
\left|1-(\sqrt{2})^{-1} 2^{-n} \delta^{m}\right|>\exp \left(-2.81 \cdot 10^{12} \log n\right),
$$

where we have used the fact that $1+\log n<1.7 \log n$ for all $n \geq 5$. Comparing of (24) and (25) gives $k<8.2 \cdot 10^{12} \log n$.

From Lemma 4 and the fact that $36.5+4 \log k+3 \log \log k<11.8 \log k$ for all $k>220$, we obtain

$$
\begin{aligned}
k & <8.2 \cdot 10^{12} \log \left(6.8 \cdot 10^{15} k^{4} \log ^{3} k\right) \\
& <8.2 \cdot 10^{12} \log (36.5+4 \log k+3 \log \log k)<9.7 \cdot 10^{13} \log k .
\end{aligned}
$$

Hence, we obtain $k<3.5 \cdot 10^{15}$, and so again from Lemma 4 we get

$$
n<4.7 \cdot 10^{82} \text { and } m<2.1 \cdot 10^{82} \text {. }
$$


Let $\Lambda_{2}:=m \log \delta-n \log 2-\log (\sqrt{2})$. By a similar method to show the inequality (23), one can see that $0<\left|\Lambda_{2}\right|<\frac{2.2}{2^{k / 2}}<2.2 \exp (-0.34 k)$ holds for all $k>220$.

Now, we will apply Lemma 3 . The inequality (26) implies that we can take $X_{0}:=4.7 \cdot 10^{82}$. Further, we can choose

$$
\begin{gathered}
c:=2.2, \quad \rho:=0.34, \quad \psi:=-\frac{\log (\sqrt{2})}{\log \delta}, \\
\vartheta:=\frac{\log 2}{\log \delta}, \quad \vartheta_{1}:=\log 2, \quad \vartheta_{2}:=-\log \delta, \quad \beta:=\log (\sqrt{2}) .
\end{gathered}
$$

With the help of Maple, we find that $q_{163} \approx 4.14 \cdot 10^{83}$ satisfies the hypotheses of Lemma 3 . Furthermore, according to Lemma 3 we obtain $k<618$.

With this new upper bound on $k$, we get from Lemma 4

$$
n<2 \cdot 10^{29} \text { and } m<8.4 \cdot 10^{28} \text {. }
$$

Applying again Lemma 3 with $X_{0}:=2 \cdot 10^{29}$ and

$$
q_{60}:=2089037648971932599649375001624
$$

in this time, we obtain $k<216$, which contradicts our assumption that $k>220$. Hence, we have shown that there are no solutions $(n, k, m)$ to equation (1) with $k>220$.

Thus, the Theorem 1 is proved.

\section{$4 \quad k$-Fibonacci Lucas-balancing numbers}

This section is devoted to prove Theorem 2. The proof of Theorem 2 is similar to that of Theorem 1. For the sake of completeness, we will give some details.

\subsection{An inequality for $n$ and $m$ in terms of $k$}

Since $F_{1}^{(k)}=F_{2}^{(k)}=1=C_{0}$, then we may assume that $n \geq 3$. For $3 \leq n \leq k+1$, we have $F_{n}^{(k)}=2^{n-2}$, but $C_{m}$ is an odd number for all $m \geq 0$, thus we deduce that the Diophantine equation (2) has no solution when $3 \leq n \leq k+1$. From now, we suppose that $n \geq k+2$.

By relations (14), (11) and equation (2) we have

$$
\alpha^{n-2} \leq \delta^{m} \quad \text { and } \quad \delta^{m-1} \leq \alpha^{n-1},
$$

hence we get

$$
(n-2)\left(\frac{\log \alpha}{\log \delta}\right) \leq m \leq(n-1)\left(\frac{\log \alpha}{\log \delta}\right)+1 .
$$

Using the fact that $3 / 2<\alpha<2$ for all $k \geq 2$ (see (12)), we deduce that

$$
0.2 n-0.5<m<0.4 n+0.7 \text {. }
$$

Lemma 7. If $(n, k, m)$ is a solution in integers of equation (2) with $k \geq 2$ and $n \geq k+2$, then the inequalities

$$
2.4 m<n<2.4 \cdot 10^{16} k^{4} \log ^{3} k
$$

hold. 
Proof. By combining (2) with (9) and (13), we obtain

$$
\left|f_{k}(\alpha) \alpha^{n-1}-\frac{\delta^{m}}{2}\right|<\frac{1}{2}+\frac{|\beta|^{m}}{2}<2
$$

Multiplying both sides by $2 \delta^{-m}$ we get

$$
\left|2 f_{k}(\alpha) \alpha^{n-1} \delta^{-m}-1\right|<2 \delta^{-m}
$$

In order to show inequality (28), we will apply Theorem 3 with the parameters $t:=3$, $\left(\eta_{1}, b_{1}\right):=\left(2 f_{k}(\alpha), 1\right),\left(\eta_{2}, b_{2}\right):=(\alpha, n-1),\left(\eta_{3}, b_{3}\right):=(\delta,-m)$, and $\Gamma_{3}:=2 f_{k}(\alpha) \alpha^{n-1} \delta^{-m}-1$.

From (29), we have that

$$
\left|\Gamma_{3}\right|<2 \delta^{-m}
$$

For this choice, the field $\mathbb{K}:=\mathbb{Q}(\alpha, \sqrt{2})$ contains $\eta_{1}, \eta_{2}, \eta_{3}$ and has $d_{\mathbb{K}} \leq 2 k$. As calculated before, we can choose $A_{2}:=2 \log 2$ and $A_{3}:=k \log \delta$.

On the other hand, using (15) and the proprieties (3) together with (4), we deduce

$$
h\left(\eta_{1}\right) \leq h(2)+h\left(f_{k}(\alpha)\right)<\log 2+\log (k+1)+\log 4<4.6 \log k
$$

for all $k \geq 2$. Thus, we obtain $\max \left\{2 k h\left(\eta_{1}\right),\left|\log \eta_{1}\right|, 0.16\right\}=9.2 k \log k:=A_{1}$. The fact that $0.4 n+0.7<n$ hold for all $n \geq 4$ and the inequality (17) imply that we may take $B:=n$.

To apply Theorem 3 , we need to show that $\Gamma_{3} \neq 0$, if it were, then

$$
f_{k}(\alpha)=\frac{\delta^{m}}{2} \alpha^{-n+1}
$$

Hence $f_{k}(\alpha)$ is an algebraic integer, which is impossible. Thus, $\Gamma_{3} \neq 0$. Therefore, after applying Theorem 3 and comparing the resulting inequality with inequality (30), we obtain

$$
m \log \delta-\log 2<1.3 \cdot 10^{13} k^{4} \log k(1+\log 2 k)(1+\log n) .
$$

Taking into account the facts $1+\log 2 k<3.5 \log k$ and $1+\log n<1.8 \log n$, which hold for $k \geq 2$ and $n \geq 4$, we deduce that

$$
m<4.65 \cdot 10^{13} k^{4} \log ^{2} k \log n .
$$

From the above inequality together with (27), it comes

$$
\frac{n}{\log n}<2.33 \cdot 10^{14} k^{4} \log ^{2} k
$$

Using (31) and (21) with $T:=2.33 \cdot 10^{14} k^{4} \log ^{2} k$ we get

$$
\begin{aligned}
n & <2\left(2.33 \cdot 10^{14} k^{4} \log ^{2} k\right) \log \left(2.33 \cdot 10^{14} k^{4} \log ^{2} k\right) \\
& <\left(4.66 \cdot 10^{14} k^{4} \log ^{2} k\right)(33.1+4 \log k+2 \log \log k)<2.4 \cdot 10^{16} k^{4} \log ^{3} k,
\end{aligned}
$$

where we have used that $33.1+4 \log k+2 \log \log k<51 \log k$, which holds for all $k \geq 2$. 


\subsection{The case $2 \leq k \leq 230$}

In this subsection, we study the case $k \in[2,230]$. We prove the following assertion.

Lemma 8. The Diophantine equation (2) has no solution when $k \in[2,230]$ and $n \geq k+2$.

Proof. Put $\Lambda_{3}=\log \left(\Gamma_{3}+1\right)=(n-1) \log \alpha-m \log \delta+\log \left(2 f_{k}(\alpha)\right)$.

Using a similar method to prove the inequality (23), we prove that

$$
0<\left|\Lambda_{3}\right|<4 \delta^{-m}<4 \exp (-1.76 m) .
$$

In Lemma 3, we fix

$$
\begin{gathered}
c:=4, \quad \delta:=1.76, \quad \psi:=\frac{\log \left(2 f_{k}(\alpha)\right)}{\log \delta}, \\
\vartheta:=\frac{\log \delta}{\log \alpha}, \quad \vartheta_{1}:=-\log \delta, \quad \vartheta_{2}:=\log \alpha, \quad \beta:=\log \left(2 f_{k}(\alpha)\right) .
\end{gathered}
$$

For each $k \in[2,230]$, we find a good approximation of $\alpha$ and a convergent $p_{\ell} / q_{\ell}$ of the continued fraction of $\vartheta$ such that $q_{\ell}>X_{0}$, where $X_{0}=\left\lfloor 2.4 \cdot 10^{16} k^{4} \log ^{3} k\right\rfloor$, which is an upper bound of $\max \{n-1, m\}$ from Lemma 7. After doing this, we use Lemma 3 on inequality (23). A computer search with Mathematica revealed that the maximum value of $\left\lfloor\frac{1}{\delta} \log \left(q^{2} c /\left|\vartheta_{2}\right| X_{0}\right)\right\rfloor$ over all $k \in[2,230]$ is $91.40 \ldots$, which according to Lemma 3 , is an upper bound on $m$. Hence, we deduce that the possible solutions $(m, n, k)$ of the equation (1) for which $k \in[2,230]$ have $m \leq 91$, therefore we use inequalities (17) to obtain $n \leq 457$.

Finally, we used Mathematica to compare $F_{n}^{(k)}$ and $C_{m}$ for the range $4 \leq n \leq 222$ and $2 \leq m \leq 44$, with $m<n / 2.4$ and checked that the only solution of the equation (1) is $3=C_{1}=F_{4}^{(2)}$.

\subsection{The case $k>230$}

In this subsection, we analyze the case $k>230$.

Lemma 9. The Diophantine equation (1) has no solution when $k>230$ and $n \geq k+2$.

Proof. For $k>230$ we have $2.4 m<n<2.4 \cdot 10^{16} k^{4} \log ^{3} k<2^{k / 2}$. By (2), (9) and (16) we obtain

$$
\left|2^{n-2}-\frac{\delta^{m}}{2}\right|<\frac{2^{n-2}}{2^{k / 2}}+\frac{1}{2}
$$

which leads to

$$
\left|1-2^{-(n-1)} \delta^{m}\right|<\frac{1.3}{2^{k / 2}}
$$

where we have used the fact $1 / 2^{n-1}<0.3 / 2^{k / 2}$, because $n \geq k+2$. We will give a lower bound to the left-hand side of inequality (32) by using Theorem 3 . We choose $t:=2,\left(\eta_{1}, b_{1}\right):=$ $(2,-n+1),\left(\eta_{2}, b_{2}\right):=(\delta, m)$. We have $\eta_{1}, \eta_{2} \in \mathbb{K}:=\mathbb{Q}(\sqrt{2})$, so $d_{\mathbb{K}}=2$. If the left-hand side of (32) is zero, then we get that $\delta^{2 m} \in \mathbb{Q}$, which is a contradiction. Thus, the left-hand side of (32) is not zero.

The fact that $m \leq n$ imply that we can choose $B:=n$. On the other hand, since $h\left(\eta_{1}\right)=\log 2, h\left(\eta_{2}\right)=(\log \delta) / 2$, it follows that

$\max \left\{2 h\left(\eta_{1}\right),\left|\log \eta_{1}\right|, 0.16\right\}=2 \log 2:=A_{1} \quad$ and $\quad \max \left\{2 h\left(\eta_{2}\right),\left|\log \eta_{2}\right|, 0.16\right\}=\log \delta:=A_{2}$. 
So, Theorem 3 tell us that

$$
\left|1-2^{-(n-1)} \delta^{m}\right|>\exp \left(-2.3 \cdot 10^{10} \log n\right),
$$

where we have used the fact that $1+\log n<1.8 \log n$ for all $n \geq 4$. Comparing (32) and (33), we obtain $k<6.7 \cdot 10^{10} \log n$.

By Lemma 7 and using the fact $37.8+4 \log k+3 \log \log k<12 \log k$ for all $k>220$, we get

$$
\begin{aligned}
k & <6.7 \cdot 10^{10} \log \left(2.4 \cdot 10^{16} k^{4} \log ^{3} k\right) \\
& <6.7 \cdot 10^{10} \log (37.8+4 \log k+3 \log \log k)<8.1 \cdot 10^{11} \log k
\end{aligned}
$$

Hence, we obtain $k<2.5 \cdot 10^{13}$. Lemma 7 imply

$$
n<2.8 \cdot 10^{74} \text { and } m<1.2 \cdot 10^{74} \text {. }
$$

Put $\Lambda_{4}=m \log \delta-(n-1) \log 2$. Using a similar method to prove the inequality (23), we show that $0<\left|\Lambda_{4}\right|<\frac{2.6}{2^{k / 2}}<2.6 \exp (-0.34 k)$ holds for all $k>210$.

We apply Lemma 1 with $c=2.6, \rho=0.34$ and $X_{0}:=2.8 \cdot 10^{74}$, which is an upper bound on $m$ by (34). Thus, from Lemma 1 we get $Y_{0}:=356.899840124 \ldots$ Let

$$
\left[a_{0}, a_{1}, a_{2}, \ldots\right]:=[0,2,1,1,5,3,2,1,22,1,5,38,1,1,1,8,1,3,7,1,5,2,5,2,2,200, \ldots]
$$

be the continued fraction expansion of $\log 2 / \log \delta$. Since $A=\max _{0 \leq 356} a_{k}=4008$, then according to Lemma 2 we have

$$
k<\frac{1}{0.34} \cdot\left(\frac{2.6 \cdot 4010 \cdot 2.8 \cdot 10^{74}}{\log \delta}\right)<530 .
$$

With this new upper bound on $k$ we get by Lemma 7 that $n<4.7 \cdot 10^{29}$ and $m<2 \cdot 10^{29}$.

We apply again Lemma 2 with $X_{0}:=4.7 \cdot 10^{29}$. Hence by Lemma 1 , we obtain $Y_{0}=142.65243 \ldots$ and $A=1014$ in this time. According to Lemma 2 it comes

$$
k<\frac{1}{0.34} \cdot\left(\frac{2.6 \cdot 1016 \cdot 4.7 \cdot 10^{29}}{\log \delta}\right)<223 \text {, }
$$

which contradicts our assumption that $k>230$. Thus, we have shown that there are no solutions $(n, k, m)$ to equation (1) with $k>230$.

Thus, the Theorem 2 is proved.

\section{References}

[1] Baker A., Davenport H. The equations $3 x^{2}-2=y^{2}$ and $8 x^{2}-7=z^{2}$. Q. J. Math. 1969, 20 (1), $129-137$. doi:10.1093/qmath/20.1.129

[2] Behera A., Panda G.K. On the Square Roots of Triangular Numbers. Fibonacci Quart. 1999, 37 (2), $98-105$.

[3] Bravo E., Bravo J.J., Luca F. Coincidences in generalized Lucas sequences. Fibonacci Quart. 2014, 52 (4), $296-306$.

[4] Bravo J.J., Gómez C.A., Luca F. Powers of two as sums of two k-Fibonacci numbers. Miskolc Math. Notes 2016, 17 (1), 85-100. doi:10.18514/MMN.2016.1505

[5] Bravo J.J., Gómez C.A., Herrera J.L. On the intersection of k-Fibonacci and Pell numbers. Bull. Korean Math. Soc. 2019, 56 (2), 535-547. doi:10.4134/BKMS.b180417 
[6] Dresden G.P.B., Du Z. A Simplified Binet Formula for k-Generalized Fibonacci Numbers. J. Integer Seq. 2014,17 (4), article 14.4.7.

[7] Dujella A., Pethó A. A generalization of a theorem of Baker and Davenport. Q. J. Math. 1998, 49 (3), $291-306$. doi:10.1093/qmathj/49.3.291

[8] Finkelstein R.P. The house problem. Amer. Math. Monthly 1965, 72 (10), 1082-1088. doi: 10.1080/00029890.1965.11970676

[9] Liptai K. Fibonacci balancing numbers. Fibonacci Quart. 2004, 42 (4), 330-340.

[10] Matveev E.M. An explicit lower bound for a homogeneous rational linear form in the logarithms of algebraic numbers. II. Izv. Math. 2000, 64 (6), 1217-1269. doi:10.1070/IM2000v064n06ABEH000314

[11] Miles E.P. Generalized Fibonacci numbers and associated matrices. Amer. Math. Monthly 1960, 67 (8), $745-752$. doi: $10.2307 / 2308649$

[12] Miller M.D. On generalized Fibonacci numbers. Amer. Math. Monthly 1971, 78 (10), 1108-1109. doi: 10.1080/00029890.1971.11992952

[13] Panda G.K. Some fascinating properties of balancing numbers. In: Proc. of 11th Intern. Conf. on Fibonacci Numbers and Their Applications, Cong. Numerantium, 2009, 194, 185-189.

[14] de Weger B.M.M. Algorithms for Diophantine equations. PhD, Eindhoven University of Technology, Eindhoven, Netherlands, 1989.

[15] Wolfram D.A. Solving generalized Fibonacci recurrences. Fibonacci Quart. 1998, 36 (2), 129-145.

Received 03.11.2020

Revised 21.12.2020

Райяне С.Е. Про збалансовані та Аюка-збалансовані иисла, що є елементами к-узагальненої послідовності Фібоначиі // Карпатські матем. публ. - 2021. - Т.13, №1. — С. 259-271.

Збалансове число $n$ і балансир $r \in$ розв' язками діофантового рівняння

$$
1+2+\cdots+(n-1)=(n+1)+(n+2)+\cdots+(n+r) .
$$

Відомо, що якщо число $n \in$ збалансованим, то $8 n^{2}+1 €$ повним квадратом, квадратний корінь

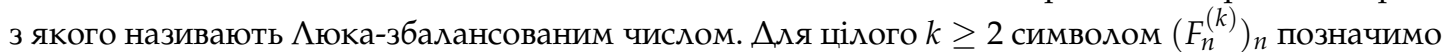
$k$-узагальнену послідовність Фібоначчі, яка починається з $0, \ldots, 0,1,1$ ( $k$ чисел), а кожне наступне число є сумою $k$ попередніх. Ми довели, шо серед елементів $k$-узагальненої послідовності Фібоначчі єлиними збалансованими числами є 1 і 6930, а Аюка-збалансованими - числа 1 і 3. Отримані нами результати узагальнюють результати з [Fibonacci Quart. 2004, 42 (4), 330-340].

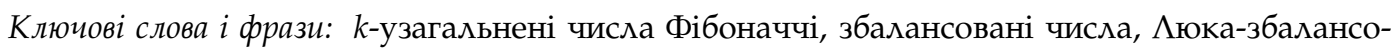
вані числа, лінійна форма в логарифмах, метод редукції. 